\title{
Gravitational microlensing as a test of stellar model atmospheres
}

\author{
1 Department of Physics and Astronomy, University of Glasgow, Glasgow, G12 8QQ, UK \\ 203 Van Allen Hall, Department of Physics and Astronomy, University of Iowa, Iowa City, IA, 52242, USA \\ 3 CNRS UMR 5572, Observatoire Midi-Pyrénées, 14 avenue E. Belin, 31400 Toulouse, France
}

Received 12 December 2001 / Accepted 2 April 2002

\begin{abstract}
We present calculations illustrating the potential of gravitational microlensing to discriminate between classical models of stellar surface brightness profiles and the recently computed "Next Generation" models of Hauschildt et al. These spherically-symmetric models include a much improved treatment of molecular lines in the outer atmospheres of cool giants - stars which are very typical sources in Galactic bulge microlensing events. We show that the microlensing signatures of intensively monitored point and fold caustic crossing events are readily able to distinguish between NextGen and the classical models, provided a photometric accuracy of $0.01 \mathrm{mag}$ is reached. This accuracy is now routinely achieved by alert networks, and hence current observations can discriminate between such model atmospheres, providing a unique insight on stellar photospheres.
\end{abstract}

Key words. gravitational lensing: stars - stars: atmospheres, fundamental parameters, imaging

\section{Introduction}

Recently gravitational microlensing has been shown to be an effective tool for stellar astrophysics, primarily through high time resolution observations of extended source events - i.e. where the lensed star cannot reasonably be approximated as a point source.

The microlensing signatures of extended sources were initially discussed in Bontz (1979), Gould (1994), Witt \& Mao (1994) and Nemiroff \& Wickramasinghe (1994) for the case of a star of uniform surface brightness, where the finite size of the star produces a significant deviation from the point source light curve. The primary motivation for the authors in the 1990's was to use extended source effects to better constrain the properties of the lens - thus distinguishing between e.g. LMC and galactic lenses.

The microlensing signatures of a non-uniform disc were first considered by Valls-Gabaud (1994), Bogdanov \& Cherepashchuk (1995), Witt (1995) and Simmons et al. (1995), who showed that the lightcurves would display a chromatic signature as the lens effectively sees a star of different radius at different wavelengths. Valls-Gabaud (1994, 1998) modelled linear, quadratic and logarithmic limb darkening, with Johnson $U$ to $K$ coefficients computed using ATLAS LTE model atmospheres from Kurucz (1994), and also predicted a spectroscopic effect, where the line profiles would change during the microlensing event, providing another probe of atmospheric structure.

Send offprint requests to: e-mail: martin@astro.gla.ac.uk
This was confirmed by Heyrovský et al. (2000) for a particular red giant model atmosphere.

Gould (2001) reviews recent observational progress in detecting non-uniform surface brightness effects. The clearest evidence of limb darkening to date comes from analysis of event MACHO 97-BLG-28 (Albrow et al. 1999). This was an example of a binary lens event with a cusp crossing - arguably the most favourable type of event for stellar astrophysics studies. The photometry and sampling coverage for this event were of sufficient quality to fit a 2-parameter limb darkening law in $V$ and $I$.

Although observations already strongly favour limb darkened atmospheres over uniform brightness models, the use of microlensing as a discriminant between limb darkened models is a relatively new subject. Rhie \& Bennett (2002) investigate the photometric signatures of 1- and 2-parameter limb darkening models from fold caustic crossing events. Albrow et al. (2001) present a detailed analysis of event OGLE 99-BUL-23, comparing the $V$ and $I$ light curves with several different model atmospheres, including for the first time a treatment of the errors in the lens parameters and their correlation with the estimated limb darkening coefficients. Notwithstanding the rigorous statistical approach of that paper, which raises the benchmark for future analyses, only linear limb darkening models were considered. The current state of the art atmosphere models are the "Next Generation" models of Hauschildt et al. (1999a,b), which include a much more detailed treatment of molecular absorption lines in the atmospheres of cool giants and consider 
spherically symmetric atmospheres, as opposed to plane parallel models used previously. The aim of this letter is to compute the microlensing signatures of typical extended source events, lensed by point and fold caustics, and to show that current observations can, in principle, already discriminate between different theoretical models - thus stimulating greater interest in microlensing observations as a test of stellar model atmospheres.

\section{PHOENIX Next Generation model atmospheres}

Computations of stellar surface brightness profiles have been carried out for several decades but until very recently were based on approximate fits to the limb darkening - i.e. the intensity, $I(\mu)$, as a function of (the cosine of) emergent angle, $\mu$. For instance a simple linear model, namely $I(\mu)=I_{0}\left[1-c_{1}(1-\mu)\right]$ was often sufficient, while detailed study of eclipsing binaries sometimes required two parameter models, such as the "square root" $I(\mu)=I_{0}\left[1-c_{2}(1-\mu)-c_{3}(1-\sqrt{\mu})\right]$ or "logarithmic" model $I(\mu)=I_{0}\left[1-c_{4}(1-\mu)-c_{5} \mu \ln \mu\right]$. Each coefficient $c_{i}$ depends on the temperature, gravity and chemical composition of the source, but the range of validity of these "laws" is often very limited (see Valls-Gabaud 1998 and references therein). For instance the square root formulation provides a good fit for hot stars, while a quadratic model fits well for cooler stars. A new non-linear formulation, using 4 parameters and valid across the entire range of effective temperatures and surface gravities was recently suggested by Claret (2000), who also made nonlinear fits to the ATLAS model atmospheres, improving upon the previous calculations of Van Hamme (1993).

By contrast, the recent PHOENIX "Next Generation" stellar atmosphere models, computed by Hauschildt et al. (1999a,b), considerably improve upon these models in several important respects, and in particular by carrying out calculations assuming spherical geometry. Moreover, the intensity calculations are based on a huge library of atomic and molecular lines, with about $2 \times 10^{8}$ molecular lines contributing to a typical giant atmosphere model at $T_{\text {eff }}=3000 \mathrm{~K}$. Claret (2000) also fitted his new non-linear limb darkening equation to some PHOENIX models.

The dramatic difference in the dependence of limb darkening on emergent angle between the ATLAS models and PHOENIX models is illustrated in Fig. 1, which shows the intensity profiles for a giant star of $T_{\text {eff }}=4250 \mathrm{~K}$ and $\log g=0.5$, in four Johnson bands: $V, R, I$ and $K$. The solid curve shows the PHOENIX profiles, while the dashed, dash-dotted and dotted curves denote the linear, logarithmic and square root models respectively. It is clear that there is a sharp decrease in the predicted intensity of the PHOENIX models close to the limb of the star, i.e. at $\mu \simeq 0.2$. This feature does not appear in ATLAS models and arises from the effects of spherical symmetry, which includes finite optical depth for rays close to the limb - a treatment which is absent from ATLAS models which utilise plane parallel atmospheres in which the
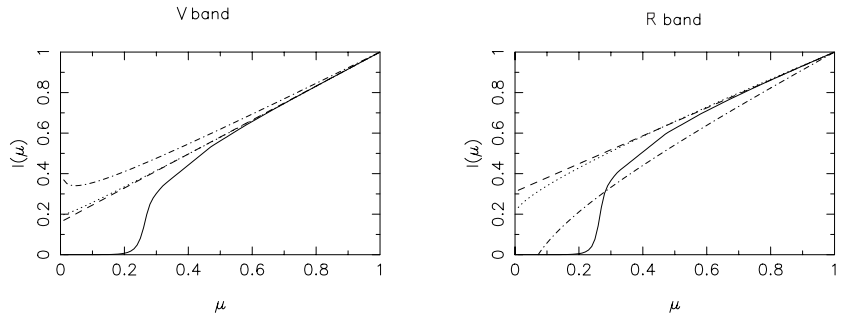

I band

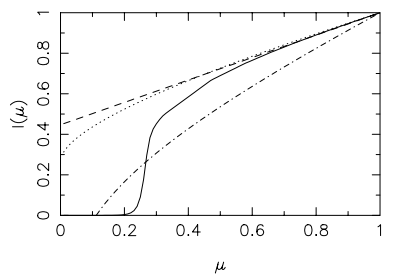

$\mathrm{K}$ band

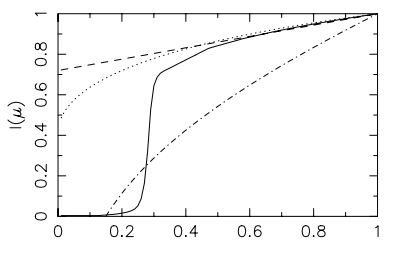

Fig. 1. Intensity profiles for a giant star of $T_{\text {eff }}=4250 \mathrm{~K}$ and $\log g=0.5$, in four Johnson passbands: $V, R, I$ and $K$. The solid curve shows the PHOENIX profiles, while the dashed, dash-dotted and dotted curves denote the ATLAS linear, logarithmic and square root models respectively.

optical depth of limb rays is still infinite and so provide significant intensity out to $\mu=0$. The question then arises: is microlensing sufficiently sensitive to detect this limb feature in the atmospheres of extended sources, and thus to test the NextGen models against real observations?

\section{Computing microlensing lightcurves}

For a point source the amplification due to a point mass lens is $A=\left(u^{2}+2\right) /\left(u \sqrt{u^{2}+4}\right)$, where $u(t)$ is the impact parameter, the projected angular separation between lens and source, measured in units of $\theta_{\mathrm{E}}$, the angular Einstein radius of the lens, defined as

$\theta_{\mathrm{E}}=\sqrt{\frac{4 G M}{c^{2}} \frac{\left(D_{\mathrm{s}}-D_{\mathrm{l}}\right)}{D_{\mathrm{s}} D_{\mathrm{l}}}}$

where $D_{1}$ and $D_{\mathrm{s}}$ are the lens-observer and source-observer separations respectively and $M$ the mass of the lens. If the impact parameter is comparable to the angular radius of the source, the point source amplification function breaks down and it becomes necessary to calculate the amplification as an integral over the source star, given by

$A_{\nu}(t)=\frac{\iint I_{\nu}(r, \theta) A(r, \theta, t) r \mathrm{~d} r \mathrm{~d} \theta}{\iint I_{\nu}(r, \theta) r \mathrm{~d} r \mathrm{~d} \theta}$.

Thus, the microlensing lightcurve contains unique information on the source surface brightness profile, $I_{\nu}(r, \theta)$, which is a far more sensitive test of the model atmosphere than its integrated value, the emergent flux.

In the case of binary lenses, the amplification function takes a more complex form, although for an extended source it is again given by an integral over the source. In this Letter we are only concerned with so-called fold caustic crossings, and so we adopt the "square-root" approximation for the amplification inside the caustic structure (Schneider et al. 1992) which is valid within a few 


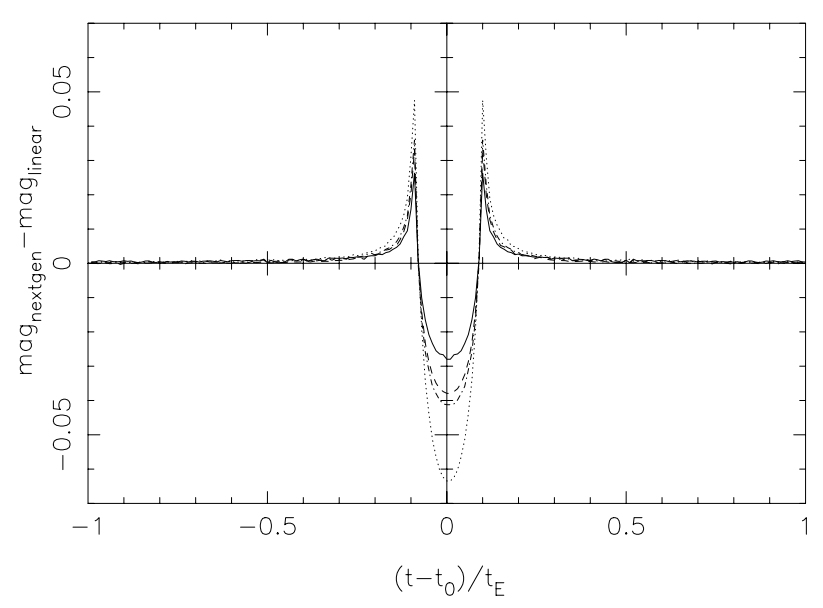

Fig. 2. A comparison of the $V, R, I$ and $K$ lightcurves produced by the transit of a point lens with minimum impact parameter $u_{0}=0.0$ of a $4250 \mathrm{~K}, \log g=0.5,0.1 \theta_{\mathrm{E}}$ source, modelled with PHOENIX and linear limb darkening. Magnitude differences are computed according to Eq. (3) in the text.

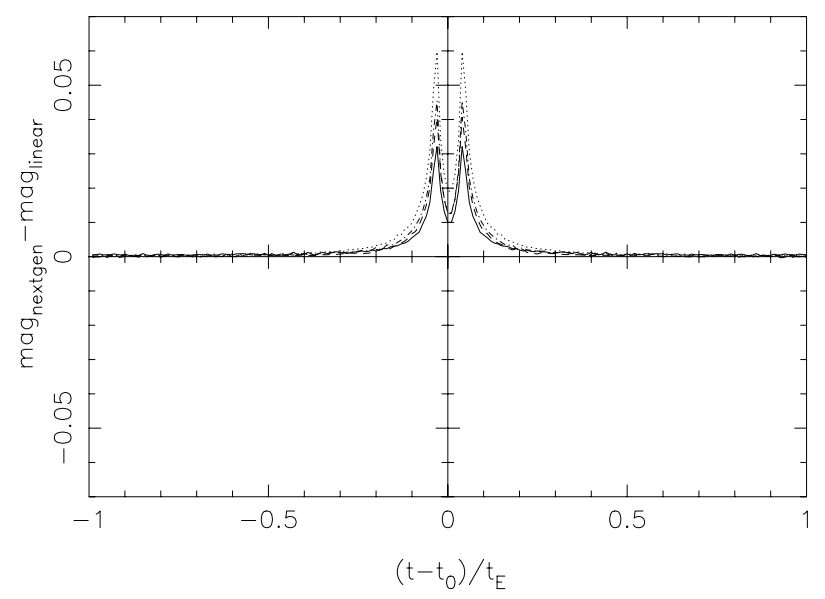

Fig. 3. A comparison of the $V, R, I$ and $K$ lightcurves as in Fig. 2, but now for an event with $u_{0}=0.09$.

angular source radii of the caustic and takes the form $A(x)=A_{0}+1 / \sqrt{x}$. Here $A_{0}$ is the total amplification of other images outside the caustic, which we assume to be constant during the caustic crossing, $x$ is the perpendicular distance in units of the angular Einstein radius of the binary lens, from an element of the source to the caustic. Note that for elements of the source outside the caustic no additional images are formed, so $A(x)=A_{0}$.

\section{Results}

Figure 2 shows a comparison between two sets of microlensing lightcurves for a point lens transit event: one set calculated using a linear limb darkening law and parameters from the ATLAS grid by Kurucz (1994) (see Valls-Gabaud 1998); and the second set calculated using the PHOENIX Next Generation models as discussed in Sect. 2. In this example we compare the models for a source with of angular radius $0.1 \theta_{\mathrm{E}}, \log g=0.5, T_{\text {eff }}=$ $4250 \mathrm{~K}$ and solar metallicity. The abscissa shows the time

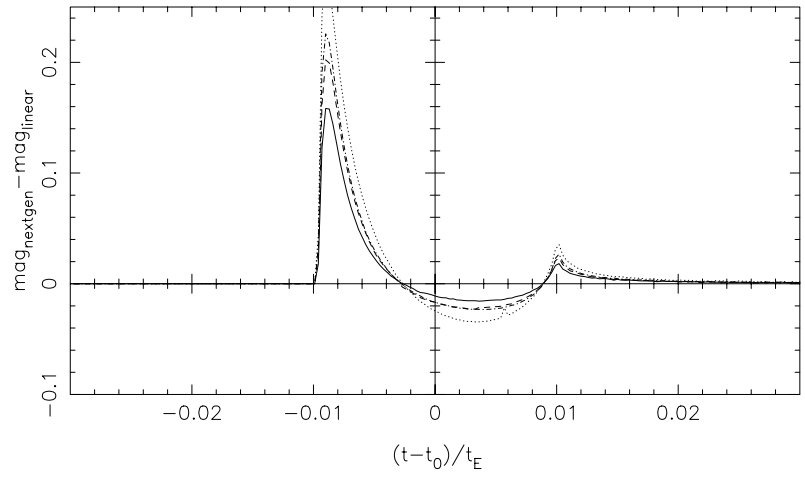

Fig. 4. A comparison of the $V, R, I$ and $K$ lightcurves produced by fold caustic crossings of $4250 \mathrm{~K}, \log g=0.5,0.1 \theta_{\mathrm{E}}$ sources with PHOENIX and linear limb darkening according to Eq. (3).

evolution of the event, in units of the Einstein crossing time, $t_{\mathrm{E}}$, of the lens - i.e. the time taken for the lens to move 1 angular Einstein radius. $t_{0}$ denotes the time at which the impact parameter is a minimum, $u_{0}$; in this example we take $u_{0}=0$ - i.e. the lens transits through the centre of the source. The ordinate shows the magnitude difference between these models, defined as

$m a g_{\text {nextgen }}-$ mag $_{\text {linear }}=2.5 \log \left(\frac{F_{\mathrm{LL}}}{F_{\mathrm{UL}}}\right)-2.5 \log \left(\frac{F_{\mathrm{LN}}}{F_{\mathrm{UN}}}\right)(3)$

where $F_{\mathrm{LL}}, F_{\mathrm{UL}}, F_{\mathrm{LN}}$ and $F_{\mathrm{UN}}$ are the lensed and unlensed linear limb darkened, and lensed and unlensed NextGen fluxes respectively. Four colour bands are used to illustrate the strong chromatic differences between the models: $V$, $R, I$ and $K$ bands, represented by straight, dashed, dashdotted and dotted lines respectively.

Two main features are immediately apparent in Fig. 2. Firstly, just before and after the lens transits the source there are positive "spikes"; these occur as the PHOENIX/NextGen model is considerably more limb darkened than the linear model close to the limb. However these spikes are very narrow. The second feature is the broad "brightening" during the central phase of the transit; this also occurs as a consequence of the strong NextGen limb darkening, which makes the source appear smaller as the lens crosses its centre, causing a larger amplification. Thus, if a source with a NextGen type atmosphere is fitted by a linear limb darkening model, the source size would be systematically underestimated.

Figure 3 compares lightcurves for the same source as Fig. 2 but with an impact parameter of $0.09 \theta_{\mathrm{E}}$, i.e. the lens just transits the source. In this case the only features are the two upward spikes, due to the considerably smaller NextGen intensity at the limb of the star. Again, however, these spikes are very narrow and around minimum impact parameter the difference between the models is much smaller, since the amplified flux is dominated by a region slightly closer to the centre of the source where there is less difference between the models (see Fig. 1 at $\mu=0.9$ ). In particular, no brightening effect is seen since the lens does not cross the central region of the source. 
The amplitude of the effect is, however, similar: at least $0.01 \mathrm{mag}$, which is readily detectable.

Figure 4 compares the NextGen and linear models during a fold caustic crossing event, shown from when the source is 3 source radii outside to 3 source radii inside the caustic structure. Here the source has $T_{\text {eff }}=4250 \mathrm{~K}$, $\log g=0.5$ and solar metallicity, but now has an angular radius of $0.01 \theta_{\mathrm{E}}-$ a more realistic value for e.g. typical Bulge events, which results in larger amplification changes. The most striking feature in Fig. 4 is the large spike as the source begins to enter the caustic; here the flux is dominated by the small region of photosphere very close to the limb and directly beneath the caustic - the surface brightness of which varies dramatically between the two models. As the event proceeds the magnitude difference becomes much smaller, particularly in mid-transit when the limb differences between the models are diluted by the larger intensity from the central part of the source. However, we again see a broad "brightening" effect as in Fig. 2, due to the extreme NextGen limb darkening producing an apparently smaller source. As the trailing limb crosses the caustic we see only a small peak since there is already considerable amplification from the rest of the photosphere. In practice, of course, we would expect to observe these events in reverse: it is easier to predict when a source will exit a caustic than when it will enter!

\section{Conclusions}

Figures 2-4 clearly suggest that it is within the capabilities of current intensive microlensing monitoring programs to discriminate between the chosen atmosphere models from a well parameterised lightcurve. For a point caustic event, with e.g. timescale $t_{\mathrm{E}} \simeq 15$ days, the transits shown in Figs. 2 and 3 would last for about 3 days, with the sharp features at each limb crossing a few hours in duration. The sampling rates regularly achieved by e.g. the PLANET collaboration during recent events would easily suffice to resolve these features with a reasonable number of data points. It must be noted, of course, that since Figs. 2 and 3 are for point lens transits, the probability of such an event - and indeed of its immediate detection - is small. Given that the event is alerted during the rise phase as a potential extended source, however, the detection of the limb crossing spikes would be highly likely.

The situation for the fold caustic event is more encouraging. Again, the timescale for the caustic crossing is of the order of several hours, which is typical of "lensing anomaly" events already regularly monitored. Moreover, as pointed out previously, the chief advantage of fold caustic crossing events is the predictability of the source exiting the caustic structure, thus allowing intensive monitoring to be scheduled in advance. As the trailing limb of the star leaves the interior of the caustic one will see a dramatic difference between NextGen and the other models since it is effectively only the (strongly darkened) limb of the star which is being amplified. The signature of this limb crossing is seen in Fig. 4 to be of order $0.2 \mathrm{mag}$ in
Johnson $B, V, R$ and $I$ - which would render it very easily detectable with existing photometric precision.

To compare stellar atmosphere models using real data would involve simultaneously determining best-fit lens parameters for the event. As remarked previously, Albrow et al. (2001) have included the effects of lens parameter errors in estimating limb darkening coefficients. We will carry out a similar numerical study, using realistic simulated observations, in future work and at a more advanced stage one must also consider the possibility of spots further effecting the lightcurve (see Hendry et al. 2002). It seems clear, however, that the magnitude of the difference between NextGen and ATLAS models is eminently detectable - even allowing for uncertainties in the fitted lens parameters. This is an essential step in the determination of fundamental stellar parameters. For instance, high accuracy measures of stellar radii via eclipsing binary light curves rely critically on these model atmospheres (e.g. Orosz \& Hauschildt 2000) as do interferometric measures (e.g. Davis et al. 2000; Wittkowski et al. 2001). Thanks to microlensing we have now almost direct access to the distribution of intensity across a source, and hence we can probe stellar photospheres with unprecedented detail.

\section{References}

Albrow, M. D., Beaulieu, J. P., Caldwell, J. A. R., et al. 1999, ApJ, 522, 1011

Albrow, M. D., An, A., Beaulieu, J. P., et al. 2001, ApJ, 549, 759

Bogdanov, M. V., \& Cherepashchuk, M. 1995, Ast. Lett., 21, 505

Bontz, R. J. 1979 ApJ, 233, 402

Claret, A. 2000, A\&A, 363, 1081

Davis, J., Tango, W., \& Booth, A. J. 2000, MNRAS, 318, 387

Gould, A. 1994, ApJ, 421, L71

Gould, A. 2001, PASP, 113, 903

Hauschildt, P. H., Allard, F., \& Baron, E. 1999a, ApJ, 512, 377

Hauschildt, P. H., Allard, F., Ferguson, J., Baron, E., \& Alexander, D. R. 1999b, ApJ, 525, 871

Hendry, M. A., Bryce, H. M., \& Valls-Gabaud, D. 2002, MNRAS, in press

Heyrovský, D., Sasselov, H., \& Loeb, A. 2000, ApJ, 543, 406

Kurucz, R. L. 1994, Atlas CDROMs

email: kurucz@cfa.harvard.edu

Nemiroff, R. J., \& Wickramasinghe, W. 1994, ApJ, 424, 21

Orosz, J. A., \& Hauschildt, P. H. 2000, A\&A, 364, 265

Rhie, S. H., \& Bennett, D. P. 2002, ApJ, in press [astro-ph/9912050]

Schneider, P., Ehlers, J., \& Falco, E. E., 1992, Gravitational Lenses (Springer-Verlag Berlin)

Simmons, J. F. L., Willis, J. P., \& Newsam, A. M. 1995, A\&A, 293, L46

Valls-Gabaud, D. 1994, in Large Scale Structures in the Universe, ed. J. Mücket, S. Gottlober, \& V. Muller (Singapore: World Scientific), 326

Valls-Gabaud, D. 1998, MNRAS, 294, 747

Van Hamme, W. 1993, AJ, 106, 2096

Witt, H. J., \& Mao, S. 1994, ApJ, 430, 505

Witt, H. J. 1995, ApJ, 449, 42

Wittkowski, M., Hummel, C. A., Johnston, K. J., et al. 2001, A\&A, 377, 981 